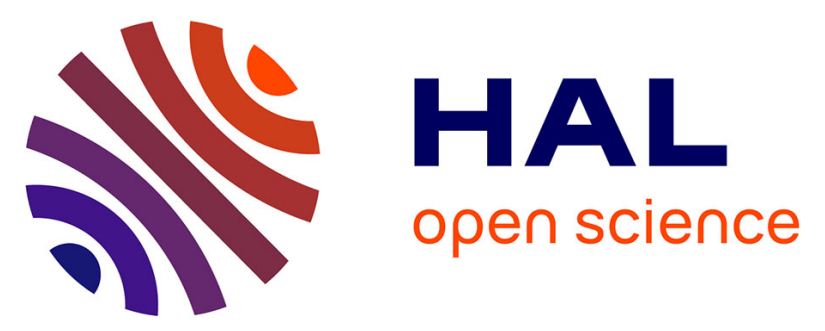

\title{
Evidence of the Key Role of Metal-Molecule Bonding in Metal-Molecule-Metal Transport Experiments
}

\author{
Lionel Patrone, Serge Palacin, Julienne Charlier, Franck Armand,
} Jean-Philippe Bourgoin, Hao Tang, Sebastien Gauthier

\section{- To cite this version:}

Lionel Patrone, Serge Palacin, Julienne Charlier, Franck Armand, Jean-Philippe Bourgoin, et al.. Evidence of the Key Role of Metal-Molecule Bonding in Metal-Molecule-Metal Transport Experiments. Physical Review Letters, 2003, 91, pp.96802. 10.1103/PhysRevLett.91.096802 . cea-01056956

\section{HAL Id: cea-01056956 https://hal-cea.archives-ouvertes.fr/cea-01056956}

Submitted on 23 Apr 2018

HAL is a multi-disciplinary open access archive for the deposit and dissemination of scientific research documents, whether they are published or not. The documents may come from teaching and research institutions in France or abroad, or from public or private research centers.
L'archive ouverte pluridisciplinaire HAL, est destinée au dépôt et à la diffusion de documents scientifiques de niveau recherche, publiés ou non, émanant des établissements d'enseignement et de recherche français ou étrangers, des laboratoires publics ou privés. 


\title{
Evidence of the Key Role of Metal-Molecule Bonding in Metal-Molecule-Metal Transport Experiments
}

\author{
L. Patrone, ${ }^{1, *}$ S. Palacin, ${ }^{1}$ J. Charlier, ${ }^{1}$ F. Armand,${ }^{1}$ J. P. Bourgoin,${ }^{1}$ H. Tang, ${ }^{2}$ and S. Gauthier ${ }^{2}$ \\ ${ }^{1}$ CEA/DSM/DRECAM CE/Saclay, 91191 Gif-sur-Yvette CEDEX, France \\ ${ }^{2}$ CEMES-CNRS, 29 rue J. Marvig, BP 4347, 31055 Toulouse CEDEX 4, France
}

(Received 20 February 2003; published 29 August 2003)

\begin{abstract}
The transport properties of two oligothiophene derivatives, that differ only by the chemical group coupling to gold, are compared. It is shown that the role of the coupling group in the transport properties can be decoupled from that of the conjugated body of the molecules and that Se is a better electronic coupling group than S. These results are accounted for semiquantitatively within the frame of the scattering theory of transport, using results from ultraviolet photoemission spectroscopy experiments as inputs for the position in energy of the molecular orbitals with respect to the Fermi level of the electrodes.
\end{abstract}

\section{DOI: 10.1103/PhysRevLett.91.096802}

Molecular electronics has seen very significant advances during the past five years. This includes the first measurements of single molecule transport, and circuits (see [1]). Understanding in depth the transport in metalmolecule-metal (MMM) junctions is a central problem that has given rise to the development of increasingly complex models [2-10]. An outcome of the scattering models, developed thus far in the Landauer picture, is the description of the low bias conductance of MMM junctions as $G=G_{0} e^{-\gamma L}$, with $G_{0}$ standing for the contact conductance defined by the molecule-metal coupling and $\gamma$ a decay constant function of the molecular structure ([11] and references therein). This exponential decay has been verified in a recent series of experiments [12-14].

The exact contribution from the contact conductance has been much less investigated thus far. Indeed, only a few theoretical and experimental studies are found in the literature that are aimed at comparing the electronic coupling strengths provided by different coupling groups. Today, the thiol group is the most commonly used link to gold [15], with only a few experimental studies made on alternative coupling groups $(\mathrm{O}, \mathrm{CN}, \mathrm{Se}$, fluoranthene, $\mathrm{Te})$ $[13,16,17]$. In a study of the barrier heights of S-, Se-, and Te-coupled molecular wires made by means of the nanopore technique, it was found that the barrier height is maximal with $\mathrm{Se}, \mathrm{S}$ and Te being lower and comparable. That conclusion, unexpected given the higher metallic character of Se compared to S, was, respectively, theoretically supported in [18] and contradicted in $[19,20]$.

In a recent study, we addressed experimentally the role of the coupling group and showed unambiguously that Se provides a more efficient electronic coupling compared to $\mathrm{S}$ [21,22]. These experiments used a scanning tunneling microscope (STM) both in air and in ultrahigh vacuum to investigate thiol and selenol terminated terthiophene molecules - T3 ( $\alpha, \omega$-bisacetylthio-terthiophene) and Se3 ( $\alpha, \omega$-bisacetylseleno-terthiophene), respectively inserted in a dodecanethiol (DT) self-assembled mono-
PACS numbers: 73.40.Cg, 73.40.Gk, 73.63.Rt, 85.65.+h

layer (SAM) on a gold surface. The insertion of single molecules into a SAM of alkanethiolate on gold was initiated by Weiss and co-workers [23]. It proved useful for the investigation of the transport properties of single conjugated molecules by STM [14,24-26]. The conclusion that $\mathrm{Se}$ is a better coupling group compared to $\mathrm{S}$ stemmed from the observation of a larger apparent height of the Se3 molecules above the DT layer $\left(\Delta h_{\mathrm{Se} 3}\right)$ compared to T3 $\left(\Delta h_{\mathrm{T} 3}\right)$, whatever the STM conditions used. The large $\Delta h_{\mathrm{Se} 3}$ (typically $3.3 \AA$ ) and $\Delta h_{\mathrm{T} 3}$ (typically $2 \AA$ ) values resulted from the conductance of the goldSe3(or T3)-tip junction being larger than that of the goldDT-tip one. The bias dependence we observed for $\Delta h_{\mathrm{Se} 3}$ and $\Delta h_{\mathrm{T} 3}$ (Fig. 5 of [22]) is a clear-cut proof that this height is related to the electronic transport properties of the molecule and not to a pure geometrical effect.

In the present Letter, we experimentally and theoretically investigate the details of the contribution of the coupling group to the contact conductance $G_{0}$ and show for the first time that the transport in MMM junctions can be simulated by an appropriate combination of experimental measurements and simple semiempirical techniques.

First, we checked by density functional theory calculations (Becke-3-Lee-Yang-Parr approximation as implemented in GAUSSIAN 98 [27]) that the two molecules isolated in vacuum have quite a similar electronic structure. In particular, their calculated ionization potentials differ by less than $1 \%$, revealing the absence of a significant difference between the two molecules when isolated in vacuum. The electronic density representation of the orbitals confirms this similarity (see supplementary material [28]). This suggests to interpret our STM results as a direct consequence of a difference in adsorption of Se3 and T3 onto gold due to their different coupling groups.

We used ultraviolet photoelectron spectroscopy (UPS) for investigating the details of the adsorption of the two molecules $[19,29]$. SAMs of pure T3 or Se3 were prepared onto gold substrates and the corresponding high resolution UPS spectra were recorded using a pass energy 
of $2 \mathrm{eV}$ and a takeoff angle of $90^{\circ}$. The integrated sampling depth was approximately $3 \mathrm{~nm}$. All spectra were referenced to the Fermi level $\left(E_{F}\right)$ of the bare metal surface obtained after ionic etching. The SAMs are thin enough to avoid any charge effect. Figure 1 shows the UPS spectra of the two molecules with details of the Fermi region. They are quite similar with peaks located around $1.5,2.5,4,6.5-7.5$, and $10 \mathrm{eV}$.

Beside the peaks at $2.5,4$, and $6.5 \mathrm{eV}$ that have some contribution from the $5 d$ orbitals of the gold substrate [30], the T3 and Se3 spectra show a good similarity with that of thick terthiophene [31] which exhibits peaks located at $2.5,4,6.5,8$, and $10 \mathrm{eV}$, each of them corresponding to more than one molecular orbital. Noticeably, the peak obtained at the lowest energy corresponds to the highest occupied molecular orbital (HOMO) and molecular orbitals of terthiophene with a pronounced $\pi$ character. Such orbitals are expected to play a role in the transport through a MMM junction. The similarity of the T3 and Se3 spectra with that of terthiophene, except for the appearance of a small peak at low energy, is a strong indication that the electronic structure of the adsorbed molecule core remains largely undisturbed by the adsorption onto the metallic surface, as predicted in [32]. This is further confirmed by the good qualitative agreement between the experimental and simulated UPS spectra of the molecules shown in Fig. 2. The simulation procedure is detailed in supplementary material [28].

Importantly, tiny but significant differences nevertheless exist between T3 and Se3 SAMs spectra (see Fig. 1): In particular, the two peaks at lower energy are shifted towards higher energies by about $0.2 \mathrm{eV}$ in the case of $\mathrm{T} 3$ compared to Se3. Conversely, the higher energy parts of the spectra are almost identical. It stems from the difference that $E_{F}-E_{\text {Hомо }}$ is smaller by about $0.2 \mathrm{eV}$ in the case

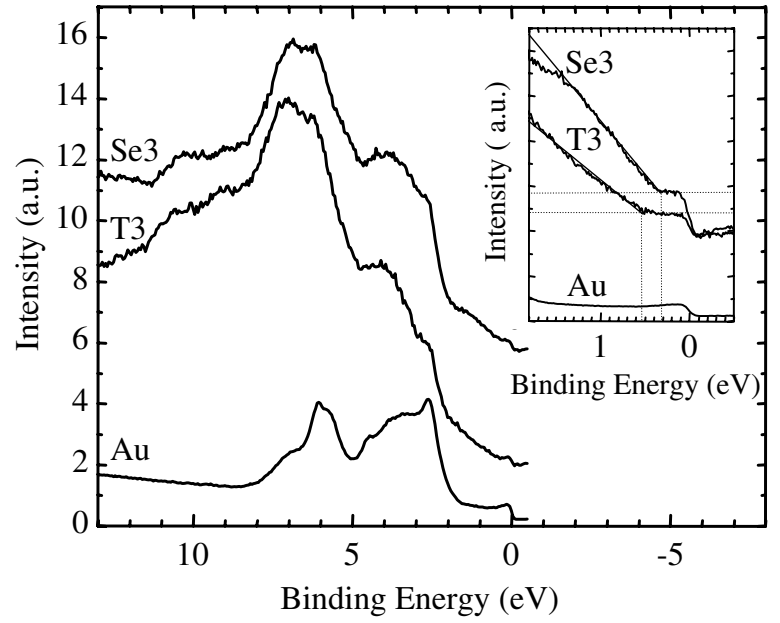

FIG. 1. UPS spectra of T3 and Se3 SAMs on gold recorded with HeII excitation line $(40.8 \mathrm{eV})$. The spectrum of gold after ion milling the organic monolayers is shown for comparison (Au). The T3 and Se3 spectra have been shifted vertically for clarity. Inset: Zoom on the Fermi energy region. of Se 3 compared to T3. This finding is consistent with the trends discussed in Ref. [19].

We conclude from this analysis that the adsorption on gold does not disturb significantly the electronic structure of the molecules but that the difference between Au-S and $\mathrm{Au}-\mathrm{Se}$ bonds shows up in the smaller $E_{F}-E_{\mathrm{HOMO}}$ value of $\mathrm{Se} 3$ compared to $\mathrm{T} 3$. This difference is crucial, pertains only to the difference in the adsorption behavior, and explains the transport difference as shown below.

We first calculated the transport properties of the goldmolecule-gold junction by the elastic scattering quantum chemistry technique (ESQC) [2]. This semiempirical technique based on an extended Hückel model has proven to quantitatively account for the low bias conductance of single adsorbed molecules as probed by STM [33]. The calculated multichannel transmission coefficient $T(E)$ of an electron at a given energy $E$ is shown in Fig. 3(a) [28]. The linear conductance $G$ of the metal-molecule-metal junction is then determined using the Landauer formula,

$$
G=\frac{2 e^{2}}{h} T\left(E_{F}\right)
$$

where $E_{F}$ is the Fermi energy of the electrodes [34]. The prediction for $G$ thus depends drastically on the value of $T$ at $E_{F}$. Note that the spectra in Fig. 3(a) are calculated with a relative energy scale due to the known inability of the Hückel technique to provide absolute values for energies.

For a given height of the STM tip, the spectra for T3 and Se3 are quite similar in shape. This was expected from the previously discussed similarity of the investigated molecules. Upon adsorption, the molecular levels are broadened and shifted by the molecule-metal electronic coupling. We note a slightly larger broadening

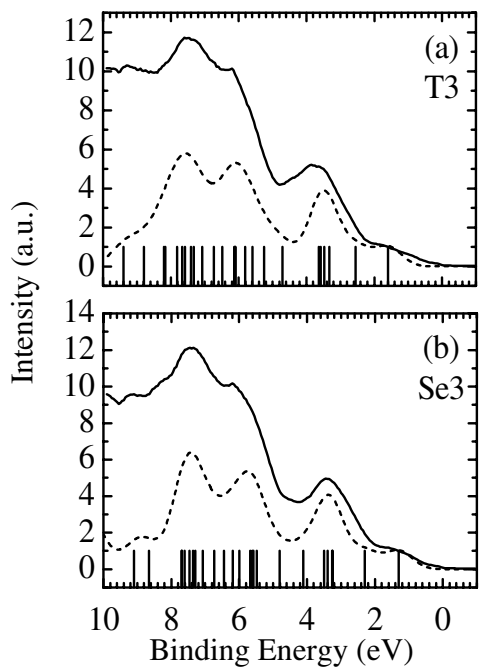

FIG. 2. Experimental and simulated HeI (21.1 eV) UPS spectra for (a) T3 and (b) Se3 SAMs on gold. At the bottom of each graph are plotted the energies corresponding to the HartreeFock molecular orbitals of isolated T3 and Se3 molecules. The simulated spectra (dashed line) are calculated as described in EPAPS. 

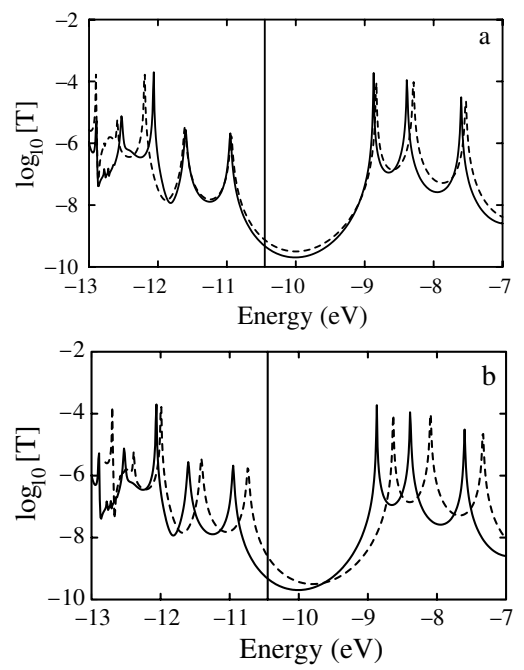

FIG. 3. Transmission spectrum $T(E)$ for a given height of the tip above T3 (solid line) and Se3 (dashed line), (a) without and (b) with adjustment of the molecular orbital energy with respect to the Fermi level of the gold electrodes (vertical line).

of the Se3 levels compared to those of T3 $(33 / 28 \mathrm{meV}$ FWHM for the HOMO levels of Se3/T3, respectively). This cannot explain alone the difference in transport properties which can only result from a difference in the shift upon adsorption, i.e., the relative position of the molecular orbital energies of the adsorbed T3 and Se3 molecules compared to the gold $E_{F}$ position. A key point for calculating the conductance or the transport in a MMM is thus to adjust this relative energy with respect to $E_{F}$. We proceed as follows. We adjust the relative energy scale of the spectra so that the $E_{F}-E_{\mathrm{HOMO}}$ value fits the $E_{F}-E_{\text {HOMO }}$ value obtained from the UPS, i.e., $0.3 \mathrm{eV}$ for $\mathrm{Se} 3$ and $0.5 \mathrm{eV}$ for T3 [35]. By so doing, it clearly appears that the zero bias conductance of a MMM junction involving T3 is lower than that of a junction involving Se3.

From these calculations, we can obtain an estimate of $\Delta h_{\mathrm{Se} 3}$ and $\Delta h_{\mathrm{T} 3}$. More precisely, we calculated both for $\mathrm{Se} 3$ and $\mathrm{T} 3$ the $T(E)$ function for various heights of the tip above the molecule. We then make use of Eq. (2) to get an estimate of the current for a given bias:

$$
I(V)=\frac{e}{\pi \hbar} \int_{-\infty}^{+\infty} T(E, V)\left[f\left(E-\mu_{1}\right)-f\left(E-\mu_{2}\right)\right] d E,
$$

where $\mu_{1,2}$ is the chemical potential of the electrode 1,2 , $f(\varepsilon)$ denotes the Fermi function, and $T(E, V)$ is the transmission spectrum for given energy and bias. Following $[4,36]$, we make the crude approximation that $T(E, V)=$ $T(E-\mathrm{eV} / 2,0)$, where $T(E, 0)$ is the transmission spectrum calculated at zero bias. This treatment allows us to obtain the current versus tip-substrate distance curves of Fig. 4. From this plot, we deduce the tip-substrate distance for a given setpoint current in the experiment. In this figure is plotted the range of current (1-60 pA) that can be used experimentally to probe DT layers without modifying them to a large extent as well as the corresponding expected tip-substrate distance for DT layers. This area (marked DT in Fig. 4) has been estimated based on $[25,37,38,39]$. We considered the following points: The minimum physical thickness of the DT layer is $14 \AA$. We assume conservatively that the tip is in contact with the monolayer for a setpoint current of $60 \mathrm{pA}$, the value at which we lose the molecular resolution. We then draw a line of slope $1 \AA^{-1}$ corresponding to the typical slope in vacuum. This gives the lower bound of the tip-substrate distance. Similarly, we draw the upper bound starting from the highest tip-surface distance reported in the literature for a $1 \mathrm{pA}$ setpoint current and using the same $1 \AA^{-1}$ slope. We note that this estimation of the tipsurface distance is reasonable and in good agreement with, for example, the ellipsometric thickness of the DT layer and various estimates of the altitude at which the tip flies over the DT layer. From the plots of Fig. 4, we can thus estimate $\Delta h_{\mathrm{Se} 3}$ and $\Delta h_{\mathrm{T} 3}$ for various setpoint currents. These values are gathered in Table I.

The qualitative agreement with the experimental values is quite reasonable, validating the principle of our interpretation. We have tested the robustness of our interpretation against variation of the bias voltage. We obtain the correct trends for the bias voltage; i.e., the observed increase of the apparent height of T3 and Se3 with increasing bias is reproduced (Fig. 5 of [22]). However, our model is too crude to account quantitatively for this evolution: It underestimates it, predicting a $\Delta h_{\mathrm{T} 3}-\Delta h_{\mathrm{Se} 3}$ difference almost independent of bias. We tested this model on other types of molecular wires based on oligophenylene-vinylene backbones and found also a good qualitative agreement [40].

It should be kept in mind that the calculated values are estimates relying on a number of approximations that must be corrected to get a quantitative agreement. This includes more realistically taking into account the bias

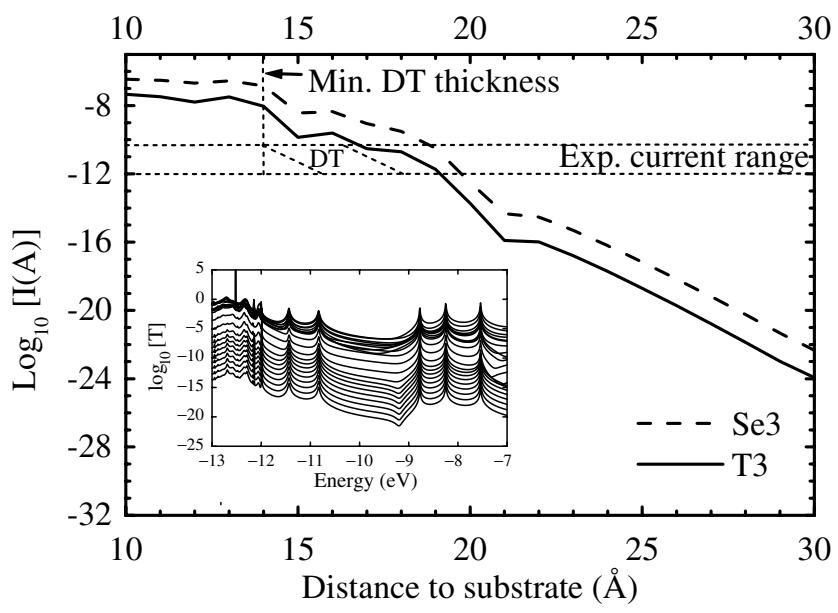

FIG. 4. Current versus distance curve obtained by integrating the $T(E)$ curves (shown in the inset for T3) calculated for different tip-surface separations. An estimate range of position of the tip above the DT layer is reported in the graph allowing one to calculate $\Delta H_{\mathrm{T} 3}$ and $\Delta H_{\mathrm{Se} 3}$. 
TABLE I. Calculated and experimental apparent heights for $\mathrm{T} 3$ and $\mathrm{Se} 3$.

\begin{tabular}{lcc}
\hline \hline Current $(\mathrm{pA})$ & $\Delta h_{\mathrm{T} 3}(\AA)$ & $\Delta h_{\mathrm{Se} 3}(\AA)$ \\
\hline Theory: 60 & $1.5 \pm 1$ & $3.5 \pm 1$ \\
Theory: 1 & $2 \pm 1$ & $2.8 \pm 1$ \\
Experiment: 1.9 pA [21] & 2 & 3.3 \\
\hline \hline
\end{tabular}

effect on the molecular energies [41], or improving the description of the contact for example. We also notice that we obtain a better agreement when we consider that the voltage drop is symmetrical at both ends of the molecule compared to the case where the voltage drop is asymmetrical (larger on the tip side where the coupling is smaller). Though such a phenomenon has already been reported [36], its origin remains unknown and the subject of investigation.

In this Letter, we have presented a number of important findings. We have shown that varying the coupling group in MMM junction is enough to give rise to a substantial difference in transport. By comparing two molecules that differ only by the coupling group and have highly similar molecular orbitals, we have directly verified for the first time the role of the coupling in the formula $G=G_{0} e^{-\gamma L}$ which separates the role of the contact from that of the conjugated part of the molecule. We have understood the origin of the electrical transport difference in MMM junctions of $\mathrm{T} 3$ and $\mathrm{Se} 3$ using UPS characterization and shown that it arises from the $E_{F}-E_{\mathrm{HOMO}}$ value being larger in the case of T3 compared to Se3. Finally, we have simulated the experiment by ESQC calculation with a good qualitative agreement. This work demonstrates that the transport in MMM junctions can be reliably simulated by an appropriate combination of experimental determination of attainable quantities and simple semiempirical techniques. We believe that this simple procedure can be used to predict the transport differences in families of related molecules.

This work was funded by the European Community within the "Nanomol," IST-1999-12603 project. Dr. B. Michel and IBM Zürich are also thanked for their kind donation of a STM that was used in this work. We also thank Dr. J. Cornil, Dr. H. Ness, and Dr. C. Bureau for helpful discussions.

*Present address: L2MP-UMR CNRS 6137, ISEM, Place G. Pompidou, 83000 Toulon, France.

[1] S. N. Yaliraki, M. A. Ratner, and P. Hanggi, Chem. Phys. 281, 1 (2002).

[2] P. Sautet and C. Joachim, Chem. Phys. Lett. 185, 23 (1989).

[3] V. Mujica, M. Kemp, and M. A. Ratner, J. Chem. Phys. 101, 6856 (1994).

[4] S. Datta et al., Phys. Rev. Lett. 79, 2530 (1997).
[5] S. N. Yaliraki and M. A. Ratner, J. Chem. Phys. 109, 5036 (1998).

[6] E. G. Emberly and G. Kirczenow, Phys. Rev. B 58, 10911 (1998).

[7] M. Di Ventra, S. T. Pantelides, and N. D. Lang, Phys. Rev. Lett. 84, 979 (2000).

[8] J. Heurich et al., Phys. Rev. Lett. 88, 256803 (2002).

[9] J. J. Palacios et al., Phys. Rev. B 66, 035322 (2002).

[10] M. Brandbyge et al., Phys. Rev. B 65, 165401 (2002).

[11] J.-P. Bourgoin, in Interacting Electrons in Nanostructures, edited by H.S. Schoeller and R. Haug (Springer-Verlag, Berlin, 2001).

[12] H. Sakaguchi et al., Appl. Phys. Lett. 79, 3708 (2001).

[13] V. Langlais et al., Phys. Rev. Lett. 83, 2809 (1999).

[14] T. Ishida et al., J. Phys. Chem. B 106, 5886 (2002).

[15] J. M. Tour et al., J. Am. Chem. Soc. 117, 9529 (1995).

[16] S. Hong et al., Superlattices Microstruct. 28, 289 (2000).

[17] W. A. Reinerth et al., Nanotechnology 9, 246 (1998).

[18] J. M. Seminario et al., J. Am. Chem. Soc. 121, 411 (1999).

[19] S. N. Yaliraki, M. Kemp, and M. A. Ratner, J. Am. Chem. Soc. 121, 3428 (1999).

[20] M. Di Ventra, N. D. Lang, and S.T. Pantelides, Chem. Phys. 281, 189 (2002).

[21] L. Patrone et al., Chem. Phys. 281, 325 (2002).

[22] L. Patrone, S. Palacin, and J. P. Bourgoin, Appl. Surf. Sci. 212-213, 446 (2003).

[23] L. A. Bumm et al., Science 271, 1705 (1996).

[24] C. Kergueris et al., Nanotechnology 10, 8 (1999).

[25] M. T. Cygan et al., J. Am. Chem. Soc. 120, 2721 (1998).

[26] G. Leatherman et al., J. Phys. Chem. B 103, 4006 (1999).

[27] M. J. Frisch et al., Gaussian Inc., Pittsburgh PA, 2001.

[28] See EPAPS Document No. E-PRLTAO-91-064332 for the details of the calculation of the multichannel transmission coefficient $T(E)$. A direct link to this document may be found in the online article's HTML reference section. The document may also be reached via the EPAPS homepage (http://www.aip.org/pubservs/epaps.html) or from ftp.aip.org in the directory /epaps/. See the EPAPS homepage for more information.

[29] A. J. Mäkinen et al., Appl. Phys. Lett. 78, 670 (2001).

[30] A. S. Duwez et al., J. Phys. Chem. B 104, 9029 (2000).

[31] H. Fujimoto et al., J. Chem. Phys. 92, 4077 (1990).

[32] Y. Q. Xue, S. Datta, and M. A. Ratner, J. Chem. Phys. 115, 4292 (2001).

[33] C. Joachim et al., Phys. Rev. Lett. 74, 2102 (1995).

[34] M. Büttiker et al., Phys. Rev. B 31, 6207 (1985).

[35] These values are obtained as shown in Fig. 1 (inset) following the procedure described in [29]. Another possibility would be to consider the position of the maximum of the peak corresponding to the HOMO (which is conceptually closer to the ESQC ground principles). We favored the former solution because it generally seems to account better for the height of barriers in transport measurements.

[36] W. D. Tian et al., J. Chem. Phys. 109, 2874 (1998).

[37] L. A. Bumm et al., J. Phys. Chem. B 103, 8122 (1999).

[38] K.-A. Son et al., Phys. Rev. Lett. 86, 5357 (2001).

[39] H. Klein et al., Mater. Sci. Eng. C 19, 279 (2002).

[40] L. Patrone et al. (unpublished).

[41] P. S. Damle, A.W. Ghosh, and S. Datta, Phys. Rev. B 64, 201403 (2001). 